EFI 93-71

\title{
ASYMPTOTIC LIMITS AND SUM RULES FOR GAUGE FIELD PROPAGATORS
}

\author{
Reinhard Oehme and Wentao Xu \\ Enrico Fermi Institute and Department of Physics \\ University of Chicago \\ Chicago, Illinois 60637, USA
}

\begin{abstract}
For gauge field propagators, the asymptotic behavior is obtained in all directions of the complex $k^{2}$-plane, and for general, linear, covariant gauges. Asymptotically free theories are considered. Except for coefficients, the functional form of the leading asymptotic terms is gauge-independent. Exponents are determined exactly by one-loop expressions. Sum rules are derived, which generalize the superconvergence relations obtained in the Landau gauge.
\end{abstract}

\footnotetext{
${ }^{1}$ Work supported in part by the National Science Foundation, Grant PHY 91-23780
} 
Interesting sum rules for the structure functions of propagators can be derived on the basis of their analytic properties, together with the asymptotic behavior for large momenta as obtained with the help of the renormalization group. For systems with a limited number of matter fields, one obtains superconvergence relations for the gauge field propagator in the Landau gauge [1, 2]. These relations are of interest in connection with the problem of confinement [4, 3]. Other results are dipole representations, and information about the discontinuity of the gauge field structure functions. They indicate the existence of an approximately linear quark-antiquark potential [6, 5], and are important for understanding the structure of the theory in the state space with indefinite metric [7].

It is the purpose of this note to present results for the gauge field propagator in general, covariant, linear gauges. We obtain the asymptotic terms for large momenta, and for all directions in the complex $k^{2}$-plane. Sum rules are derived, which generalize the superconvergence relations of the Landau gauge. An important aspect of our results is the gauge-independence of the functional form of the essential asymptotic terms. Only the coefficients of these terms depend upon the gauge parameter.

We consider a non-Abelian gauge theory like QCD, with the gauge-fixing part of the Lagrangian given by $-B \cdot\left(\partial_{\mu} A^{\mu}\right)+\frac{\alpha}{2} B \cdot B$, where $B$ is the usual auxiliary field. For $\alpha \neq 0, B$ can be eliminated by $\alpha B=\left(\partial_{\mu} A^{\mu}\right)$. In order to define the structure function of the transverse gauge field propagator, we write

$$
\begin{gathered}
\int d x e^{i k x}\left\langle 0\left|T A_{a}^{\mu \nu}(x) A_{b}^{\varrho \sigma}(0)\right| 0\right\rangle=-i \delta_{a b} D\left(k^{2}+i 0\right) \\
\times\left(k^{\mu} k^{\varrho} g^{\nu \sigma}-k^{\mu} k^{\sigma} g^{\nu \varrho}+k^{\nu} k^{\sigma} g^{\mu \varrho}-k^{\nu} k^{\varrho} g^{\mu \sigma}\right)
\end{gathered}
$$

with $A^{\mu \nu} \equiv \partial^{\mu} A^{\nu}-\partial^{\nu} A^{\mu}$. We assume the general postulates of covariant gauge theories. Important are Lorentz covariance and simple spectral conditions, as formulated in references like [8, 9] for state spaces with indefinite metric. Exact Green's functions should be connected with the formal perturbation series in the coupling parameter $g$ for $g^{2} \rightarrow+0$, at least as far as the first few terms are concerned. Topological aspects of the gauge theory are not expected to influence the asymptotic behavior we consider here.

As a consequence of Lorentz covariance, and the spectral conditions mentioned above, it follows that the function $D\left(k^{2}+i 0\right)$ is the boundary value of 
an analytic function, which is regular in the cut $k^{2}$-plane, with a branch line along the positive real axis. In contrast to the situation for higher Green's functions [10], explicite use of local commutativity is not required for the two-point functions [1]. Using renormalization group methods, together with analyticity, we obtain the asymptotic behavior for $k^{2} \rightarrow \infty$ in all directions of the complex plane. We present first the essential leading terms, leaving derivation and details for later.

For the analytic structure function $D\left(k^{2}\right)$, we find for $k^{2} \rightarrow \infty$ in all directions:

$$
\begin{aligned}
-k^{2} D\left(k^{2}, \kappa^{2}, g, \alpha\right) \simeq \frac{\alpha}{\alpha_{0}}+ & C_{R}\left(g^{2}, \alpha\right)\left(-\beta_{0} \ln \frac{k^{2}}{\kappa^{2}}\right)^{-\gamma_{00} / \beta_{0}}+\cdots \\
& +\frac{\alpha}{\alpha_{0}} C_{1}\left(-\beta_{0} \ln \frac{k^{2}}{\kappa^{2}}\right)^{-1}+\cdots
\end{aligned}
$$

The corresponding asymptotic terms for the discontinuity along the positive, real $k^{2}$-axis are then given by

$$
\begin{gathered}
-k^{2} \rho\left(k^{2}, \kappa^{2}, g, \alpha\right) \simeq \frac{\gamma_{00}}{\beta_{0}} C_{R}\left(g^{2}, \alpha\right)\left(-\beta_{0} \ln \frac{k^{2}}{\left|\kappa^{2}\right|}\right)^{-\gamma_{00} / \beta_{0}-1}+\cdots \\
+\frac{\alpha}{\alpha_{0}} C_{1}\left(-\beta_{0} \ln \frac{k^{2}}{\left|\kappa^{2}\right|}\right)^{-2}+\cdots
\end{gathered}
$$

The parameters and their limitations are as follows: The function $D$ is normalized at the real point $k^{2}=\kappa^{2}<0$, where

$$
-\left.k^{2} D\left(k^{2}, \kappa^{2}, g, \alpha\right)\right|_{k^{2}=\kappa^{2}}=1 .
$$

The anomalous dimension of the gauge field is given by $\gamma\left(g^{2}, \alpha\right)=\gamma_{0}(\alpha) g^{2}+$ $\gamma_{1}(\alpha) g^{4}+\cdots, \gamma_{0}(\alpha)=\gamma_{00}+\alpha \gamma_{01}, \gamma_{1}(\alpha)=\gamma_{10}+\alpha \gamma_{11}+\alpha^{2} \gamma_{12}$, etc., and $\alpha_{0} \equiv-\gamma_{00} / \gamma_{01}$. The renormalization group function is $\beta\left(g^{2}\right)=\beta_{0} g^{4}+$ $\beta_{1} g^{6}+\cdots$. For QCD, we have $\gamma_{00}=-\left(16 \pi^{2}\right)^{-1}\left(\frac{13}{2}-\frac{2}{3} N_{F}\right), \gamma_{01}=\left(16 \pi^{2}\right)^{-1} \frac{3}{2}$, $\beta_{0}=-\left(16 \pi^{2}\right)^{-1}\left(11-\frac{2}{3} N_{F}\right), N_{F}=$ number of flavors. We assume $\beta_{0}<0$ corresponding to asymptotic freedom. Consequently, the exponent $\gamma_{00} / \beta_{0}$ in eqs. (2) and (3) varies from $13 / 22$ for $N_{F}=0$ to $1 / 10$ for $N_{F}=9$, and from $-1 / 16$ for $N_{F}=10$ to $-15 / 2$ for $N_{F}=16$. We have $0<\gamma_{00} / \beta_{0}<1$ for $N_{F} \leq 9$ and $\gamma_{00} / \beta_{0}<0$ for $10 \leq N_{F} \leq 16$; for $\gamma_{00} / \beta_{0}=1$, our relations (2) and (3) would require modifications. 
The essential asymptotic term in eqs.(2) and (3) is the one with the coefficient $C_{R}\left(g^{2}, \alpha\right)$, which is not identically zero, although there may be zero surfaces $\alpha=\alpha_{0}\left(g^{2}\right)$. In case $C_{R}$ should vanish, the $C_{1}$ - term becomes relevant. Its coefficient is given by

$$
\begin{aligned}
& C_{1}=\frac{\gamma_{1}\left(\alpha_{0}\right)}{\beta_{0}-\gamma_{00}} \quad \text { for } 0<\frac{\gamma_{00}}{\beta_{0}}<1, \\
& C_{1}=\frac{\gamma_{00}+\alpha_{0} \gamma_{11}}{\beta_{0}-\gamma_{00}} \quad \text { for } \frac{\gamma_{00}}{\beta_{0}}<0 .
\end{aligned}
$$

For $\alpha=0$, the coefficient $C_{R}$ is positive and given by

$$
\begin{aligned}
C_{R}\left(g^{2}, 0\right) & =\left(g^{2}\right)^{-\gamma_{00} / \beta_{0}} \exp \int_{g^{2}}^{0} d x \tau_{0}(x), \\
\tau_{0}(x) & \equiv \frac{\gamma(x, 0)}{\beta(x)}-\frac{\gamma_{00}}{\beta_{0} x}
\end{aligned}
$$

Eq. (6) follows from the exact solution for $\alpha=0$, with the normalization $-\kappa^{2} D\left(\kappa^{2}, \kappa^{2}, g, 0\right)=1$ [回, 2]. This solution can be written in the form

$$
-k^{2} D\left(k^{2}, \kappa^{2}, g, 0\right)=\left(\frac{\bar{g}^{2}}{g^{2}}\right)^{\gamma_{00} / \beta_{0}} \exp \int_{g^{2}}^{\bar{g}^{2}} d x \tau_{0}(x) .
$$

Here $\bar{g}^{2}=\bar{g}^{2}(u, g), u=\left|\frac{k^{2}}{\kappa^{2}}\right|$ is the effective gauge coupling, with the properties

$$
\begin{aligned}
\ln u & =\int_{g^{2}}^{\bar{g}^{2}(u, g)} d x \beta^{-1}(x), \\
\bar{g}^{2}(u, g) & \simeq 1 /\left(-\beta_{0} \ln u\right)+\cdots
\end{aligned}
$$

for $u \rightarrow \infty$, in the case of asymptotic freedom with $\beta_{0}<0$.

In general, $C_{R}\left(g^{2}, \alpha\right)$ satisfies a partial differential equation. In an approximation, where only terms linear in $\alpha$ are kept in the expression for the anomalous dimension $\gamma\left(g^{2}, \alpha\right)$, we obtain

$$
\begin{aligned}
C_{R}\left(g^{2}, \alpha\right) & =\left(g^{2}\right)^{-\gamma_{00} / \beta_{0}} \exp \left(\int_{g^{2}}^{0} d x \tau_{0}(x)\right) \\
& \times\left\{1-\frac{\alpha}{\alpha_{0}}+\frac{\alpha}{\alpha_{0}}\left(g^{2}\right)^{\gamma_{00} / \beta_{0}} \int_{g^{2}}^{0} d x x^{-\gamma_{00} / \beta_{0}} f\left(x, g^{2}\right)\right\}
\end{aligned}
$$


Here

$$
\begin{aligned}
f\left(x, g^{2}\right) & =\left\{\tau_{0}(x)+\alpha_{0} \tau_{1}(x)\right\} \exp \int_{x}^{g^{2}} d y \tau_{0}(y) \\
\tau_{1}(x) & =\bar{\gamma}_{1}(x) / \beta(x)-\gamma_{01} / \beta_{0} x \\
\gamma(x, \alpha) & =\bar{\gamma}_{0}(x)+\alpha \bar{\gamma}_{1}(x)+O\left(\alpha^{2}\right) .
\end{aligned}
$$

In eq. (9), the normalization for the full solution in the the $\alpha$-linear approximation has been used in order to fix an otherwise undetermined coefficient of $C_{R}$. This approximation consists of replacing the general anomalous dimension $\gamma\left(g^{2}, \alpha\right)$ by the $\alpha$-linear form $\bar{\gamma}_{0}\left(g^{2}\right)+\alpha \bar{\gamma}_{1}\left(g^{2}\right)$. The corresponding solution is given by

$$
\begin{aligned}
-k^{2} D\left(k^{2}, \kappa^{2}, g, \alpha\right) & =\exp \left(\int_{g^{2}}^{\bar{g}^{2}} d x \frac{\bar{\gamma}_{0}(x)}{\beta(x)}\right) \\
& \times\left\{1+\alpha \int_{g^{2}}^{\bar{g}^{2}} d x \frac{\bar{\gamma}_{1}(x)}{\beta(x)} \exp \left(-\int_{g^{2}}^{x} d y \frac{\bar{\gamma}_{0}(y)}{\beta(y)}\right)\right\},
\end{aligned}
$$

with the notation as defined in eqs. (8) and (10).

An important aspect of the leading asymptotic terms for

$$
-k^{2} D\left(k^{2}, \kappa^{2}, g, \alpha\right)-\frac{\alpha}{\alpha_{0}} \text { and } \quad-k^{2} \rho\left(k^{2}, \kappa^{2}, g, \alpha\right)
$$

is their independence of the gauge parameter $\alpha$, except for the coefficient $C_{R}\left(g^{2}, \alpha\right)$. In addition, the exponents of the logarithms in eqs. (2) and (3) are completely and exactly determined by one-loop coefficients of the anomalous dimension $\gamma\left(g^{2}, 0\right)$ and of the $\beta$-function.

In view of the asymptotic behavior of $D\left(k^{2}, \kappa^{2}, g, \alpha\right)$ as given in eq. (2), which is valid for all directions in the $k^{2}$-plane, we can write the usual unsubstracted dispersion representation

$$
D\left(k^{2}, \kappa^{2}, g, \alpha\right)=\int_{-0}^{\infty} d k^{2} \frac{\rho\left(k^{\prime 2}, \kappa^{2}, g, \alpha\right)}{k^{\prime 2}-k^{2}}
$$

We also have sufficient boundedness for the discontinuity $\rho$ in order to write a dipole representation

$$
D\left(k^{2}, \kappa^{2}, g, \alpha\right)=\int_{-0}^{\infty} d k^{\prime 2} \frac{\sigma\left(k^{\prime 2}, \kappa^{2}, g, \alpha\right)}{\left(k^{\prime 2}-k^{2}\right)^{2}},
$$




$$
\sigma\left(k^{2}, \kappa^{2}, g, \alpha\right)=\int_{-0}^{k^{2}} d k^{\prime 2} \rho\left(k^{\prime 2}, \kappa^{2}, g, \alpha\right)
$$

For $\alpha=0$, the dipole representation has been discussed in refs. [5] and [6] in connection with an approximately linear quark-antiquark potential.

Of particular interest is the situation for $0<\gamma_{00} / \beta_{0}<1$, corresponding to $N_{F} \leq 9$ in QCD. There, the function $D+\frac{\alpha}{\alpha_{0}} k^{-2}$ vanishes faster than $k^{-2}$ for $k^{2} \rightarrow \infty$, and hence we have the sum rule

$$
\int_{-0}^{\infty} d k^{2} \rho\left(k^{2}, \kappa^{2}, g, \alpha\right)=\frac{\alpha}{\alpha_{0}} .
$$

This is the generalization of the superconvergence relation [1, 2]

$$
\int_{-0}^{\infty} d k^{2} \rho\left(k^{2}, \kappa^{2}, g, 0\right)=0
$$

which was obtained previously in the Landau gauge. The relation (14) expresses the fact that the coefficient of the asymptotic term proportional to $k^{-2}$ in the representation (12) is given by $-\alpha / \alpha_{0}$. It is not valid for $\gamma_{00} / \beta_{0}<0$. The distribution aspects of sum rules like eq. (14), and of the related dispersion representations, have been discussed in refs. [1], 2].

In order to derive the asymptotic properties of the structure function $D$, we consider the renormalization group equation for the dimensionless function $R\left(k^{2} / \kappa^{2}, g, \alpha\right) \equiv-k^{2} D\left(k^{2}, \kappa^{2}, g, \alpha\right)$. We obtain

$$
R\left(\frac{k^{2}}{\kappa^{2}}, g, \alpha\right)=R\left(\frac{\kappa^{\prime 2}}{\kappa^{2}}, g, \alpha\right) R\left(\frac{k^{2}}{\kappa^{\prime 2}}, \bar{g}, \bar{\alpha}\right),
$$

where we have used the relation

$$
R^{-1}\left(\kappa^{\prime 2} / \kappa^{2}, g, \alpha\right)=Z_{3}\left(\kappa^{\prime 2} / \kappa^{2}, g, \alpha\right)
$$

which follows from the normalization condition (4): $R(1, g, \alpha)=1$ at $k^{2}=$ $\kappa^{2}<0$, and where $Z_{3}$ is the square of the conventional renormalization factor for the gauge field. Further, in eq. (15), $\bar{g}=\bar{g}\left(\frac{\kappa^{\prime 2}}{\kappa^{2}}, g\right)$ is the effective (running) gauge coupling parameter, and $\bar{\alpha}$ is given by $\bar{\alpha}=\alpha R^{-1}\left(\frac{\kappa^{\prime 2}}{\kappa^{2}}, g, \alpha\right)$. 
We use a mass independent renormalization scheme [11], which is appropriate for the study of asymptotic limits.

Let us first consider the limit $k^{2} \rightarrow-\infty$ along the negative, real $k^{2}$-axis, where $R\left(k^{2} / \kappa^{2}, g, \alpha\right)$ is analytic and real. We set $u=\left|k^{2} / \kappa^{2}\right|$ and define the function $R\left(\bar{g}^{2} ; g^{2}, \alpha\right) \equiv R(u, g, \alpha)$, with $\bar{g}(u, g)$ being the effective coupling defined in eq. (8). From eq. (15), we then obtain the differential equation

$$
\begin{array}{r}
\beta\left(\bar{g}^{2}\right) \frac{\partial R\left(\bar{g}^{2} ; g^{2}, \alpha\right)}{\partial \bar{g}^{2}}=\gamma\left(\bar{g}^{2}, \bar{\alpha}\right) R\left(\bar{g}^{2} ; g^{2}, \alpha\right), \\
\bar{\alpha}=\bar{\alpha}\left(\bar{g}^{2} ; g^{2}, \alpha\right) \equiv \alpha R^{-1}\left(\bar{g}^{2} ; g^{2}, \alpha\right) .
\end{array}
$$

For $\alpha \neq 0$, it is more convenient to work with the equation

$$
\beta\left(\bar{g}^{2}\right) \frac{\partial \bar{\alpha}}{\partial \bar{g}^{2}}=-\bar{\alpha} \gamma\left(\bar{g}^{2}, \bar{\alpha}\right)
$$

with $\bar{\alpha}$ as defined in eq. (16). From the general solution (7) of eq. (16) for $\alpha=0$, as well as the solution (11) for $\alpha \neq 0$ in the $\alpha$-linear approximation, we know that $\mathrm{R}$ and $\bar{\alpha}$ have a branch point as a function of $\bar{g}^{2}$ at $\bar{g}^{2}=0$, which is of the form $\left(\bar{g}^{2}\right)^{\xi}, \xi \equiv \gamma_{00} / \beta_{0}$. For QCD, we have $\xi=\frac{\frac{13}{2}-\frac{2}{3} N_{F}}{11-\frac{2}{3} N_{F}}$. We see that $|\xi|=n / d$ is rational, with $n$ and $d$ being relative primes. It is then convenient to uniformize the algebraic branch point by introducing $x=\left(\bar{g}^{2}\right)^{1 / d}$ as a uniformization variable.

We consider first the case $0<\xi<1$, corresponding to $N_{F} \leq 9$ for QCD. Here it is convenient to use eq. (16). We define $y(x)=\left(\bar{\alpha}-\alpha_{0}\right) x^{-n}$, and obtain the differential equation

$$
\frac{d y}{d x}=\frac{n}{\alpha_{0}} x^{n-1} y^{2}-d x^{d-n-1}\left(\alpha_{0}+x^{n} y\right) \phi\left(x^{d}, \alpha_{0}+x^{n} y\right),
$$

where

$$
\begin{aligned}
\phi\left(g^{2}, \alpha\right) & \equiv \frac{\gamma\left(g^{2}, \alpha\right)}{\beta\left(g^{2}\right)}-\frac{\gamma_{0}(\alpha)}{\beta_{0} g^{2}}=\phi_{0}(\alpha)+g^{2} \phi_{1}(\alpha)+\cdots \\
\phi_{0}(\alpha) & =\frac{\gamma_{1}(\alpha)}{\beta_{0}}-\frac{\beta_{1}}{\beta_{0}^{2}} \gamma_{0}(\alpha), \quad \text { etc. }
\end{aligned}
$$

with the definitions given below eq. (田). 
In an appropriate finite domain including $g^{2}=0$, and excluding possible, nontrivial fixed points corresponding to zeroes of $\beta\left(g^{2}\right)$, it is reasonable to assume that $\phi\left(g^{2}, \alpha\right)$ is continuously differentiable. As far as $\beta\left(g^{2}\right)$ and $\gamma\left(g^{2}, \alpha\right)$ are represented by power series expansions for $g^{2} \rightarrow+0, \phi\left(g^{2}, \alpha\right)$ is also a power series in $g^{2}$ and $\alpha$. Under these circumstances, the r.h.s. of eq. (18) satisfies the Lipschitz condition for $x=0$, and we have exactly one solution through every point $x=0, y=C$. In as far as the r.h.s. of eq. (18) is also a power series, we obtain the solution in the form of a series:

$$
\begin{aligned}
y(x)=C+ & \frac{C^{2}}{\alpha_{0}} x^{n}+C\left(\frac{\xi+1}{\xi-1} \phi_{0}\left(\alpha_{0}\right)-\alpha_{0} \phi_{0}^{\prime}\left(\alpha_{0}\right)\right) x^{d}+\cdots \\
& +\frac{\alpha_{0}}{\xi-1} \phi_{0}\left(\alpha_{0}\right) x^{d-n}+\cdots,
\end{aligned}
$$

where we have separated the terms proportional to $C$.

For the asymptotic expansion of $R\left(\bar{g}^{2} ; g^{2}, \alpha\right)$ for $\bar{g}^{2} \rightarrow+0$, eq. (20) implies

$$
\begin{aligned}
R\left(\bar{g}^{2} ; g^{2}, \alpha\right) \simeq \frac{\alpha}{\alpha_{0}}+ & C_{R}\left(\bar{g}^{2}\right)^{\xi}+C_{R} \beta_{0}^{-1}\left(\gamma_{10}-\gamma_{12} \alpha_{0}^{2}-\frac{\beta_{1}}{\beta_{0}} \gamma_{00}\right)\left(\bar{g}^{2}\right)^{\xi+1}+\cdots \\
& +\frac{\alpha}{\alpha_{0}} \frac{\gamma_{1}\left(\alpha_{0}\right)}{\beta_{0}} \frac{1}{1-\xi} \bar{g}^{2}+\cdots
\end{aligned}
$$

with $C_{R}=-C \alpha / \alpha_{0}^{2}$, and $0<\xi<1$. This formula is also valid for $C_{R}=0$. The term proportional to $C^{2}$ in eq.(20) cancels in the inversion leading to eq. (21).

For $\xi<0$, corresponding to $10 \leq N_{F} \leq 16$ for QCD, it is more convenient to use eq. (16). With $\xi=-\frac{n}{d}, n$ and $d$ being positive integers which are relative primes, we uniformize the branch point at $\bar{g}^{2}=0$ by introducing again a new variable $x$ so that $\bar{g}^{2}=x^{d}$. Then we define $z(x)=x^{n} R\left(x^{d} ; g^{2}, \alpha\right)$, and obtain the differential equation

$$
\frac{d z}{d x}=\alpha_{0} \alpha n x^{n-1}+d x^{d-1} z \phi\left(x^{d}, \frac{\alpha x^{n}}{z}\right)
$$

As long as $x^{n} z^{-1}$ remains bounded around $x=0$, we have again the Lipschitz condition satisfied and obtain the power series solution

$$
\begin{aligned}
z(x) & =C_{R}+C_{R} \phi_{0}(0) x^{d}+\cdots \\
& +\frac{\alpha}{\alpha_{0}} x^{n}+\frac{\alpha}{\alpha_{0}} \frac{d}{d+n}\left(\phi_{0}(0)+\alpha_{0} \phi_{0}^{\prime}(0)\right) x^{d+n}+\cdots
\end{aligned}
$$


with $C_{R} \neq 0$. In terms of $R\left(\bar{g}^{2} ; g^{2}, \alpha\right)$, eq. (23) leads to the asymptotic expansion for $\xi<0$ and $\bar{g}^{2} \rightarrow+0$ :

$$
\begin{gathered}
R\left(\bar{g}^{2} ; g^{2}, \alpha\right) \simeq C_{R}\left(\bar{g}^{2}\right)^{\xi}+C_{R} \frac{1}{\beta_{0}}\left(\gamma_{10}-\frac{\beta_{1}}{\beta_{0}} \gamma_{00}\right)\left(\bar{g}^{2}\right)^{\xi+1}+\cdots \\
+\frac{\alpha}{\alpha_{0}}+\frac{\alpha}{\alpha_{0}} \frac{\left(\gamma_{10}+\alpha_{0} \gamma_{11}\right)}{\beta_{0}(1-\xi)} \bar{g}^{2}+\cdots
\end{gathered}
$$

For $C_{R}=0$, we cannot use eq. (22), but obtain the asymptotic expression directly from eqs. (20) and (21) :

$$
R\left(\bar{g}^{2} ; g^{2}, \alpha\right) \simeq \frac{\alpha}{\alpha_{0}}+\frac{\alpha}{\alpha_{0}} \frac{\gamma_{1}\left(\alpha_{0}\right)}{\beta_{0}} \frac{1}{1+|\xi|} \bar{g}^{2}+\cdots .
$$

This relation corresponds to eq. (21) with $C_{R}=0$ and $\xi<0$.

With eqs. (21) and (24), we have obtained the asymptotic expressions for $R\left(\frac{k^{2}}{\kappa^{2}}, g, \alpha\right)$ in the limit $k^{2} \rightarrow-\infty$ along the real axis, provided we can use $\bar{g}^{2}(u, g) \simeq-1 /\left(\beta_{0} \ln u\right)+\cdots$. It remains to consider the limit $k^{2} \rightarrow \infty$ in all directions of the complex $k^{2}$-plane. We return to eq. (15). Setting $\kappa^{\prime 2}=-\left|k^{2}\right|$, we find, with $\kappa^{2}<0$ and $k^{2}=-\left|k^{2}\right| e^{i \varphi}$ for all $|\varphi| \leq \pi$ :

$$
R\left(\frac{k^{2}}{\kappa^{2}}, g, \alpha\right)=R\left(\left|\frac{k^{2}}{\kappa^{2}}\right|, g, \alpha\right) R\left(e^{i \varphi}, \bar{g}, \bar{\alpha}\right) .
$$

Here $\bar{g}=\bar{g}\left(\left|\frac{k^{2}}{\kappa^{2}}\right|, g\right)$ and $\bar{\alpha}=\alpha R^{-1}\left(\left|\frac{k^{2}}{\kappa^{2}}\right|, g, \alpha\right)$. For $\beta_{0}<0$, the effective coupling $\bar{g}^{2}$ vanishes for $\left|\frac{k^{2}}{\kappa^{2}}\right| \rightarrow \infty$, and $\bar{\alpha}$ remains bounded in this limit, as may be seen from eqs. (21) and (24). Because $R$ is analytic in the cut complex $k^{2}$-plane, we can then use the perturbation expansion for the structure function,

$$
R\left(\frac{k^{2}}{\kappa^{2}}, g, \alpha\right) \simeq 1+g^{2} \gamma_{0}(\alpha) \ln \left(\frac{k^{2}}{\kappa^{2}}\right)+O\left(g^{4}\right),
$$

and write for $\bar{g}^{2} \rightarrow+0$ :

$$
R\left(e^{i \varphi}, \bar{g}, \bar{\alpha}\right) \simeq 1+\bar{g}^{2} \gamma_{0}(\bar{\alpha}) i \varphi+O\left(\bar{g}^{4}\right) .
$$

Eq. (26) expresses the asymptotic limit for $k^{2} \rightarrow \infty$ in all directions in terms of the limit along the negative real $k^{2}$-axis. With eqs. (26), (28), (21) and (24), we finally obtain the limits given in eqs. (2) and (3). 
A priori, the coefficients $C$ or $C_{R}$ appearing in the solutions of the nonlinear, ordinary differential equations are undetermined constants. However, because of the normalization condition $R\left(g^{2} ; g^{2}, \alpha\right)=1$ or $\bar{\alpha}\left(g^{2} ; g^{2}, \alpha\right)=\alpha$, the coefficients become functions of $g^{2}$ and $\alpha$, satisfying partial differential equations in these variables. For $C_{R}\left(g^{2}, \alpha\right)$, we find the equation

$$
\begin{aligned}
C_{R}\left(g^{2}, \alpha\right) & =R\left(g^{\prime 2} ; g^{2}, \alpha\right) C_{R}\left(g^{\prime 2}, \alpha^{\prime}\right) \\
\alpha^{\prime} & =\alpha R^{-1}\left(g^{\prime 2} ; g^{2}, \alpha\right)
\end{aligned}
$$

and the corresponding differential equation is:

$$
\beta\left(g^{2}\right) \frac{\partial C_{R}}{\partial g^{2}}=\alpha \gamma\left(g^{2}, \alpha\right) \frac{\partial C_{R}}{\partial \alpha}-\gamma\left(g^{2}, \alpha\right) C_{R}
$$

For $\alpha=0$, and in the $\alpha$-linear approximation, we have given $C_{R}$ in eqs. (6) and (9), which satisfy eq. (30) with $\alpha=0$ and $\gamma\left(g^{2}, \alpha\right)=\bar{\gamma}_{0}\left(g^{2}\right)+\alpha \bar{\gamma}_{1}\left(g^{2}\right)$ respectively.

Several of the results presented in this paper have been obtained by one

of us (R.O.) in collaboration with W. Zimmermann [12]. It is a pleasure to thank Wolfhart Zimmermann for his contribution and for many discussions.

\section{References}

[1] R. Oehme and W. Zimmermann, Phys. Rev. D21 (1980) 475, 1661.

[2] R. Oehme, Phys. Lett. B252 (1990) 641.

[3] K. Nishijima, in Symmetry in Nature, Festschrift for Luigi A. Radiacati di Brozolo (Scuola Normale Superiore, Pisa, 1989) pp. 627-655.

[4] R. Oehme, Phys. Rev. D42 (1990) 4209.

[5] R. Oehme, Phys. Lett. B232 (1989) 498.

[6] K. Nishijima, Prog. Theor. Phys. 77 (1987) 1035. 
[7] R. Oehme, Mod. Phys. Lett. A8 (1993) 1533;

$\pi N$ - Newsletter No. 7 (1992) 1.

[8] T. Kugo and I. Ojima, Prog. Theor. Phys. Suppl. No. 66 (1978) 1;

N. Nakanishi and I. Ojima, Covariant Operator Formalism of Gauge Theories and Quantum Gravity (World Scientific, Singapore, 1990) p. 382.

[9] F. Strocchi, Comm. Math. Phys. 56 (1977) 57;

Phys. Rev. D17 (1978) 2010.

[10] H.J. Bremermann, R. Oehme and J.G. Taylor, Phys. Rev. 109 (1958) 2178.

[11] S. Weinberg, Phys. Rev. D8 (1973) 3497;

G. t'Hooft, Nucl. Phys. B61 (1973) 455;

J.C. Collins and A.J. MacFarlane, Phys. Rev. D10 (1974) 1201.

[12] R. Oehme and W. Zimmermann, (1983) unpublished. 